\title{
QED corrections to Higgs boson decay into four leptons at the LHC
}

\author{
Carlo Michel Carloni Calame, Guido Montagna and Oreste Nicrosini \\ INFN Sezione di Pavia and Dipartimento di Fisica Nucleare e Teorica \\ v. A. Bassi 6, I-27100 Pavia, Italy \\ E-mail: carlo.carloni.calame@pv.infn.it, guido.montagna@pv.infn.it, \\ oreste.nicrosini@pv.infn.it
}

\section{Mauro Moretti}

Dipartimento di Fisica Università di Ferrara and INFN Sezione di Ferrara

v. Paradiso 12, I-44100 Ferrara, Italy

E-mail: mauro.moretti@fe.infn.it

\section{Fulvio Piccinini*}

INFN Sezione di Pavia and Dipartimento di Fisica Nucleare e Teorica

v. A. Bassi 6, I-27100 Pavia, Italy

E-mail: fulvio.piccinini@pv.infn.it

\section{Antonio D. Polosa}

INFN Sezione di Roma, Italy

E-mail: antonio.polosa@cern.ch

At the LHC a precise measurement of the Higgs boson mass (if discovered), at the level of 0.1$1 \%$, will be possible through the channel $g g \rightarrow H \rightarrow 4 l$ for a wide range of Higgs mass values. To match such an accuracy, the systematic effects induced by QED corrections need to be investigated. In the present study the calculation of $\mathscr{O}(\alpha)$ and higher order QED corrections is illustrated as well as their impact on the Higgs mass determination, once realistic event selections for charged leptons and photons are considered.

International Europhysics Conference on High Energy Physics

July 21st - 27th 2005

Lisboa, Portugal

\footnotetext{
* Speaker.
} 


\section{Introduction}

Assuming that the Higgs boson will be discovered at the LHC, a measurement of its mass, for a large range of values, with a relative experimental precision of $0.1-1 \%$ will be possible, by combining ATLAS and CMS measurements and for an integrated luminosity of $300 \mathrm{fb}^{-1}$ per experiment [1]. To match such an accuracy, the impact of QED radiative corrections on the Higgs mass determination through the process $g g \rightarrow H \rightarrow 4 l\left(4 l=4 e, 4 \mu, e^{+} e^{-} \mu^{+} \mu^{-}\right)$must be evaluated. To this end exact $\mathscr{O}(\alpha)$ and higher-order QED corrections are calculated and their effect on the Higgs mass extraction is evaluated according to realistic event selections. Even if the electromagnetic corrections affect only the final state, the effects of the production process have to be considered, because the typical event selections applied on leptons are not Lorentz invariant. The complete process $p p \rightarrow H \rightarrow 4 l(\gamma)$ has been simulated in the narrow width approximation, well justified for low Higgs masses, thus allowing to factorize the production and decay processes. The obtained preliminary results show that QED radiation effects should be carefully considered in view of the expected precision at the LHC.

\section{The calculation}

QED radiative corrections are calculated on top of exact tree-level matrix elements for the decay $H \rightarrow Z^{(*)} Z^{(*)} \rightarrow 4 l$, which consist of one diagram for the final state $e^{+} e^{-} \mu^{+} \mu^{-}$and two diagrams for equal flavour leptonic pairs. Since the tree-level is mediated by electrically neutral $Z$-bosons, QED corrections can be calculated in a gauge invariant way as a subset of the complete electroweak corrections [2], namely neglecting $W$-boson exchange contributions. Two complementary approaches have been adopted: the former is based on the parton shower technique, in the realization of Ref. [3], allowing to calculate QED corrections in the leading logarithmic approximation both at $\mathscr{O}(\alpha)$ and to all orders; the latter relies upon a pure $\mathscr{O}(\alpha)$ perturbative calculation. While in the first case the correction is completely factorized over the tree-level, in the diagrammatic calculation some care has to be devoted to the treatment of the $Z$-boson width in the virtual and real corrections in order to safely control the infrared (IR) cancellations. As for any one-loop calculation, the expression for the decay width, fully differential over the final state, can be written as $(d \Gamma)_{B}+(d \Gamma)_{V}+(d \Gamma)_{R}$, where the subscripts stand for Born (B), Virtual (V) and Real (R) contributions. The IR divergence is regularized by means of a small photon mass $\lambda$. The real corrections are calculated analytically in soft approximation for $\lambda \leq E_{\gamma} \leq k_{0}$, (where $k_{0}$ is the softhard separator) and by means of exact matrix elements for $E_{\gamma}>k_{0}$, with finite fermion masses and $\lambda=0$. The real hard photon emission diagrams have been calculated analytically with FORM [4] and cross-checked with ALPHA [5]. The virtual corrections consist of vertex and self-energy diagrams which are symbolically written in terms of Passarino-Veltman form factors [6] and evaluated numerically with LoopTools [7]. In addition, also box and pentagon diagrams are present. The pentagon diagrams are reduced, with the help of FORM [4], to combinations of four-point form factors by means of the techniques introduced in Ref. [8], in order to avoid numerical instabilities due to vanishing Gram determinants. The method has already been successfully used for the calculation of the $\mathscr{O}(\alpha)$ electroweak corrections to $e^{+} e^{-} \rightarrow 4$ fermions, where also six-point functions are involved [9]. Adopting the approximation of vanishing fermion masses whenever possible in 
the virtual corrections and performing the calculation in the 't Hooft-Feynman gauge $\xi=1$, the involved five-point functions are at most of rank two. An additional complication is due to the presence of the unstable $Z$ bosons. In order to avoid singularities in the phase space, the introduction of the $Z$ width is required, which could spoil the IR cancellation between virtual and real corrections. In fact the IR divergences contained in the non-factorizable five-point diagrams are cancelled by the interference between real (tree-level) radiation from different external legs. A solution is given by the "complex mass scheme" (introduced in Ref. [10] for lowest order processes and generalized for one-loop calculation in Ref. [9]), where the $Z$ mass is shifted on the complex plane with fixed width $M_{Z}^{2} \rightarrow M_{Z}^{2}-i \Gamma_{Z} M_{Z}$, both in tree-level and in loop diagrams and the couplings become complex quantities, in order to respect the Ward identities. Considering that self-energies and vertex corrections, neglecting terms of $\mathscr{O}\left(m_{f}^{2} / Q^{2}\right)$, are already factorized over the tree-level, and that with complex $M_{Z}$ the IR singularity can be factorized over the tree-level also for five-point diagrams, the $\mathscr{O}(\alpha)$ QED corrected Higgs partial width can be written as:

$$
(d \Gamma)_{B} \times\left(1+\delta_{V}^{\mathrm{fact}}+\delta_{V}^{5-I R}\right)+(d \Gamma)_{R}+\left[(d \Gamma)_{V}^{\mathrm{nf}}-(d \Gamma)_{B} \times \delta_{V}^{5-I R}\right],
$$

where $\delta_{V}^{\text {fact }}$ refers to the contribution of self-energies and vertices, $\delta_{V}^{5-I R}$ refers to the IR scalar three-point functions representing the IR part of the five-point functions and $(d \Gamma)_{V}^{\mathrm{nf}}$ is the complete non-factorizable contribution. By construction, the IR divergence has been factorized over the tree-level allowing for a consistent IR cancellation with the real part $(d \Gamma)_{R}$ and the remainder $\left[(d \Gamma)_{V}^{\mathrm{nf}}-(d \Gamma)_{B} \times \delta_{V}^{5-I R}\right]$ is free of divergence. While the numerical evaluation of two- and threepoint scalar functions with complex masses is performed with LoopTools v2.2, the four-point scalar functions is reduced to unidimensional integrals which are evaluated by means of the adaptive integration package CUBA [11]. With the same numerical algorithm several checks have been performed of the two- and three-point functions with complex masses obtained with LoopTools (which uses the formulae implemented in $\mathrm{FF}$ [12]), finding up-to-digit agreement.

In order to estimate realistically the impact of QED corrections on the Higgs mass determination, the complete production and decay process $p p \rightarrow H \rightarrow 4 l(n \gamma)$ has to be simulated, considering typical realistic event selections. Since for a Higgs mass value below the real Z-pair production threshold the total width is very small compared to its mass, the narrow width approximation is adequate. In this approximation the calculation can be split in on-shell production $\times$ decay. A Monte Carlo code has been developed, based on the event generator ALPGEN [13] for Higgs production and an orginal library $\mathrm{H} 24 \mathrm{~F}$ for the decay into four leptons with QED radiative corrections taking into account realistic event selections for charged leptons and photons. The additional approximation of neglecting the Higgs transverse momentum is assumed in the present study. A more detailed investigation will be presented elsewhere.

\section{Effect of QED corrections on Higgs mass determination}

To quantify the shift induced by QED corrections on Higgs mass determination, binned $\chi^{2}$ fits to the (four leptons) invariant mass distribution have been performed, following the strategy described in detail in Ref. [14]. In order to be close to the experimental situation, lepton identification requirements and kinematical cuts, as well as uncertainties in the energy and transverse momentum measurements of the photons/leptons in the detector, have been implemented according to Ref. [1]. 


\begin{tabular}{|c|c|c|}
\hline Process & $\left|\Delta(Q E D)^{(\alpha)}\right|$ & $\left|\Delta(Q E D)^{(\exp )}-\Delta(Q E D)^{(\alpha)}\right|$ \\
\hline$e^{+} e^{-} e^{+} e^{-}$ & $160 \mathrm{MeV}$ & $\leq 20 \mathrm{MeV}$ \\
\hline$e^{+} e^{-} \mu^{+} \mu^{-}$ & $340 \mathrm{MeV}$ & $\leq 50 \mathrm{MeV}$ \\
\hline$\mu^{+} \mu^{-} \mu^{+} \mu^{-}$ & $600 \mathrm{MeV}$ & $\sim 100 \mathrm{MeV}$ \\
\hline
\end{tabular}

Table 1: The Higgs mass shifts due to $\mathscr{O}(\alpha)$ and higher-order QED corrections to $g g \rightarrow H \rightarrow 4 l$ for the three different lepton final states and for a Higgs mass of $130 \mathrm{GeV}$. The uncertainty on $\left|\Delta(Q E D)^{(\alpha)}\right|$ is of the order of $20 \mathrm{MeV}$.

The results of this preliminary analysis are given in Tab. 1, showing the Higgs mass shifts due to $\mathscr{O}(\alpha)$ (second column) and higher-order (third column) corrections for the three possible four lepton decays and for a Higgs mass of $130 \mathrm{GeV}$. It can be seen that the mass shift due to multiple photon radiation is, as a rule of thumb, of the order of $10 \%$ of that caused by one photon emission and, therefore, non-negligible in view of the expected precision at the LHC. Furthermore, it has been preliminarily observed that, at the present level of accuracy of the investigation, exact $\mathscr{O}(\alpha)$ and leading logarithmic $\mathscr{O}(\alpha)$ QED corrections induce the same mass shifts. A more detailed analysis, as well as a presentation of further numerical results, is left to a future work.

\section{References}

[1] S. Asai et al., SN-ATLAS-2003-024; M. Düehrssen, ATL-PHYS-2003-030; S. Abdullin et al., CMS Note 2003/33; ATLAS Technical Design Report ATLAS-TDR 15, CERN/LHCC 99-15.

[2] A. Bredenstein, A. Denner, S. Dittmaier and M.M. Weber, talk given at RADCOR 2005, Shonan Village, Japan, 2-7 October 2005.

[3] C.M. Carloni Calame, C. Lunardini, G. Montagna, O.Nicrosini and F. Piccinini, Nucl. Phys. B584 (2000) 459; C.M. Carloni Calame, Phys. Lett., B520 (2001) 16.

[4] J.A.M. Vermaseren, arXiv:[math-ph/0010025].

[5] F. Caravaglios and M. Moretti, Phys. Lett. B358 (1995) 332.

[6] G. Passarino and M.J. Veltman, Nucl. Phys. B160 (1979) 151.

[7] T. Hahn and M. Perez-Victoria, Comp. Phys. Commun. 118 (1999) 153; T. Hahn, talk given at RADCOR 2005, Shonan Village, Japan, 2-7 October 2005.

[8] A. Denner and S. Dittmaier, Nucl. Phys. B658 (2003) 175.

[9] A. Denner, S. Dittmaier, M. Roth and L.H. Wieders, Phys. Lett. B612 (2005) 223; arXiv:[hep-ph/0505042]; A. Denner and S. Dittmaier, arXiv:[hep-ph/0509141].

[10] A. Denner, S. Dittmaier, M. Roth and D. Wackeroth, Nucl. Phys. B560 (1999) 33.

[11] T. Hahn, Comput. Phys. Commun. 168 (2005) 78.

[12] G.J. van Oldenborgh and J.A.M. Vermaseren, Z. Phys. C46 (1990) 425.

[13] M.L. Mangano, M. Moretti, F. Piccinini, R. Pittau and A.D. Polosa, JHEP 0307 (2003) 001.

[14] C.M. Carloni Calame, G. Montagna, O.Nicrosini and M. Treccani, Phys. Rev. D69 (2004) 037301; JHEP 05 (2005) 019. 